\title{
Zeta Potential Determination from Molecular
}

\section{Simulations}

\author{
Denys Biriukov, ${ }^{+}$Pavel Fibich,, Milan Předota ${ }^{+, *}$ \\ 'Institute of Physics, Faculty of Science, University of South Bohemia, Branišovská 1760, \\ 370 05, České Budějovice, Czech Republic \\ ${ }^{\ddagger}$ Department of Botany, Faculty of Science, University of South Bohemia, Branišovská 1760, \\ 370 05, České Budějovice, Czech Republic \\ ${ }^{*}$ Corresponding author: E-mail: predota@prf.jcu.cz
}

\section{Supporting Information}

Example Gromacs and LAMMPS input files can be found at

http://home.prf.jcu.cz/ predota/zeta/biriukov/ 
Table S1. Force Field Parameters Employed in This Work ${ }^{a}$

\begin{tabular}{|c|c|r|r|r|}
\hline \multirow{2}{*}{ atom } & model & $\mathrm{q}[\mathrm{e}]$ & $\sigma[\AA]$ & $\varepsilon\left[\mathrm{kJ} \mathrm{mol}^{-1}\right]$ \\
\hline \multirow{3}{*}{$\mathrm{Na}^{+}$} & ECC & +0.75 & 2.115 & 0.544284 \\
\cline { 2 - 5 } & full-rutile $^{2}$ & +1 & 2.583 & 0.4184 \\
\cline { 2 - 5 } & full-quartz & +1 & 2.15954 & 1.47545 \\
\hline \multirow{2}{*}{$\mathrm{Rb}^{+}$} & ECC $^{4}$ & +0.75 & 2.97 & 1.86231 \\
\hline \multirow{2}{*}{$\mathrm{Sr}^{2+}$} & ECC $^{4}$ & +1.5 & 2.97 & 0.4947 \\
\hline $\mathrm{Ca}^{2+}$ & ECC $^{5}$ & +1.5 & 2.6656 & 0.5072 \\
\hline \multirow{3}{*}{$\mathrm{Cl}^{-}$} & ECC $^{6}$ & -0.75 & 4.1 & 0.4928 \\
\cline { 2 - 5 } & full-rutile & -1 & 4.4401 & 0.4184 \\
\cline { 2 - 5 } & full-quartz & -1 & 4.83045 & 0.0534924 \\
\hline
\end{tabular}

${ }^{a}$ Force field parameters for rutile (110) surfaces are summarized in ref 4. Lennard-Jones parameters and original 'full' charges of quartz (101) surface atoms are summarized in ref 7, while 'ECC' charges are summarized in Table S2. Force field parameters for oxalic acid anions are summarized in Ref 8 (the ECCR-P parameterization of hydrogenoxalate has been used). 
Table S2. ECC Charges of Quartz (101) Surface Atoms ${ }^{a}$

\begin{tabular}{|r|r|c|c|c|c|c|c|}
\hline \multirow{2}{*}{$\begin{array}{c}\text { surface charge density } \\
{\left[\mathrm{C} / \mathrm{m}^{2}\right]}\end{array}$} & \multicolumn{2}{|c|}{ bulk atoms } & \multicolumn{3}{c|}{$\mathrm{SiOH}$ atoms } & \multicolumn{2}{c|}{$\mathrm{SiO}^{-}$atoms } \\
\cline { 2 - 9 } & $\mathrm{Si}_{\mathrm{s}}$ & $\mathrm{O}_{\mathrm{s}}$ & \multicolumn{1}{c|}{$\mathrm{Si}_{\mathrm{h}}$} & $\mathrm{O}_{\mathrm{h}}$ & $\mathrm{H}_{\mathrm{h}}$ & $\mathrm{Si}_{\mathrm{o}}$ & $\mathrm{O}^{-}$ \\
\hline-0.03 & 2.0598 & -1.0312 & 2.06505 & -0.9352 & 0.4218 & 1.97005 & -0.9812 \\
\hline-0.06 & 2.0588 & -1.0322 & 2.0637 & -0.9362 & 0.4208 & 1.9677 & -0.9822 \\
\hline-0.12 & 2.0566 & -1.0344 & 2.0616 & -0.9384 & 0.4186 & 1.9658 & -0.9844 \\
\hline
\end{tabular}

${ }^{a}$ The variable charge atoms are summarized here. The charges of bulk $\mathrm{SiO}_{2}$ atoms are $q\left(\mathrm{Si}_{\mathrm{B}}\right)=2.1$ and $q\left(\mathrm{O}_{\mathrm{B}}\right)=-1.05$ as in ref 7. 


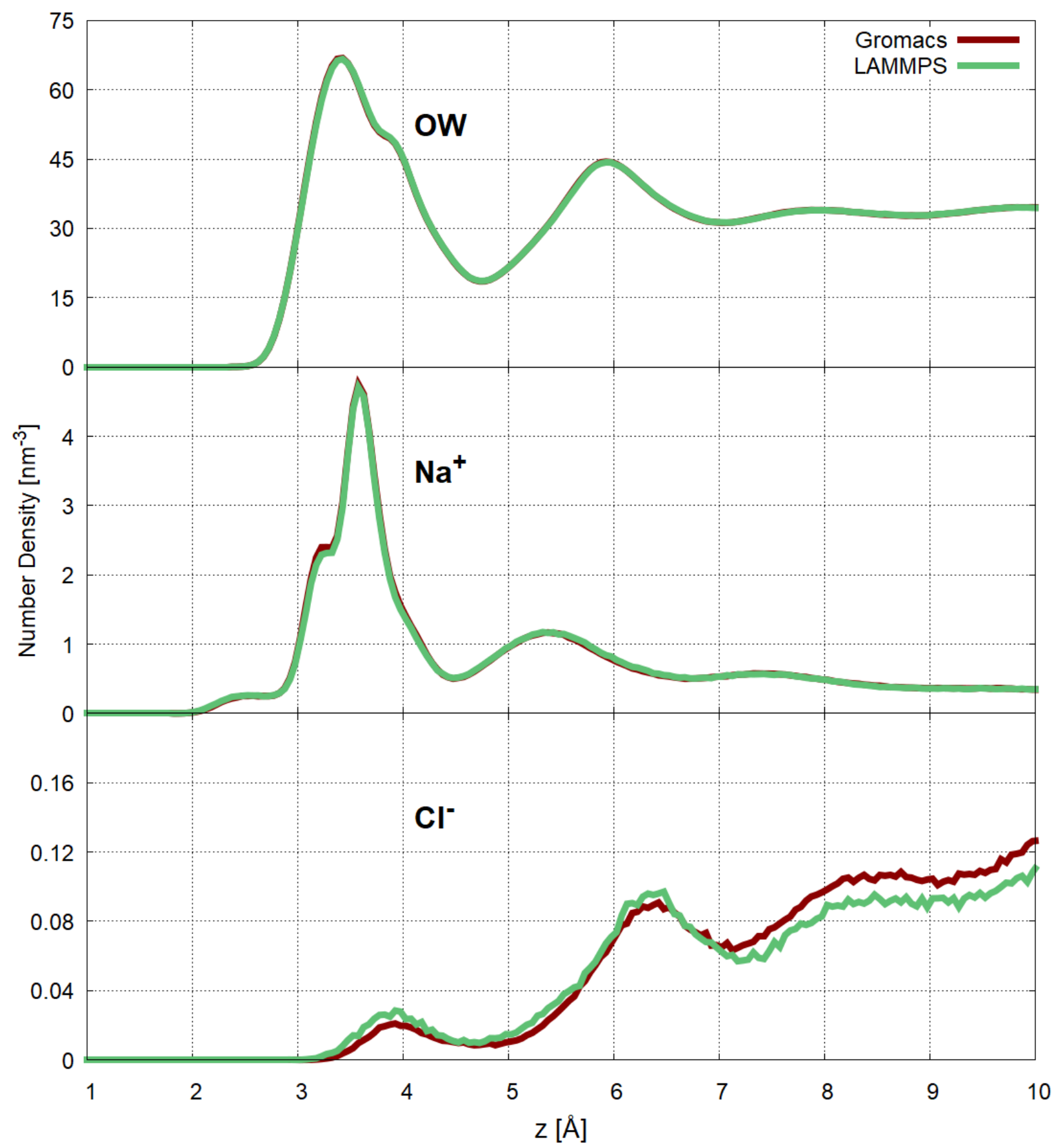

Figure S1. Axial number density profiles of water and ions of $0.33 \mathrm{M} \mathrm{NaCl}$ solution at the most negatively charged $\left(-0.12 \mathrm{C} / \mathrm{m}^{2}\right)$ quartz $(101)$ surface from the equilibrium $\mathrm{MD}$ simulations performed in different software packages. Note different vertical scales. 


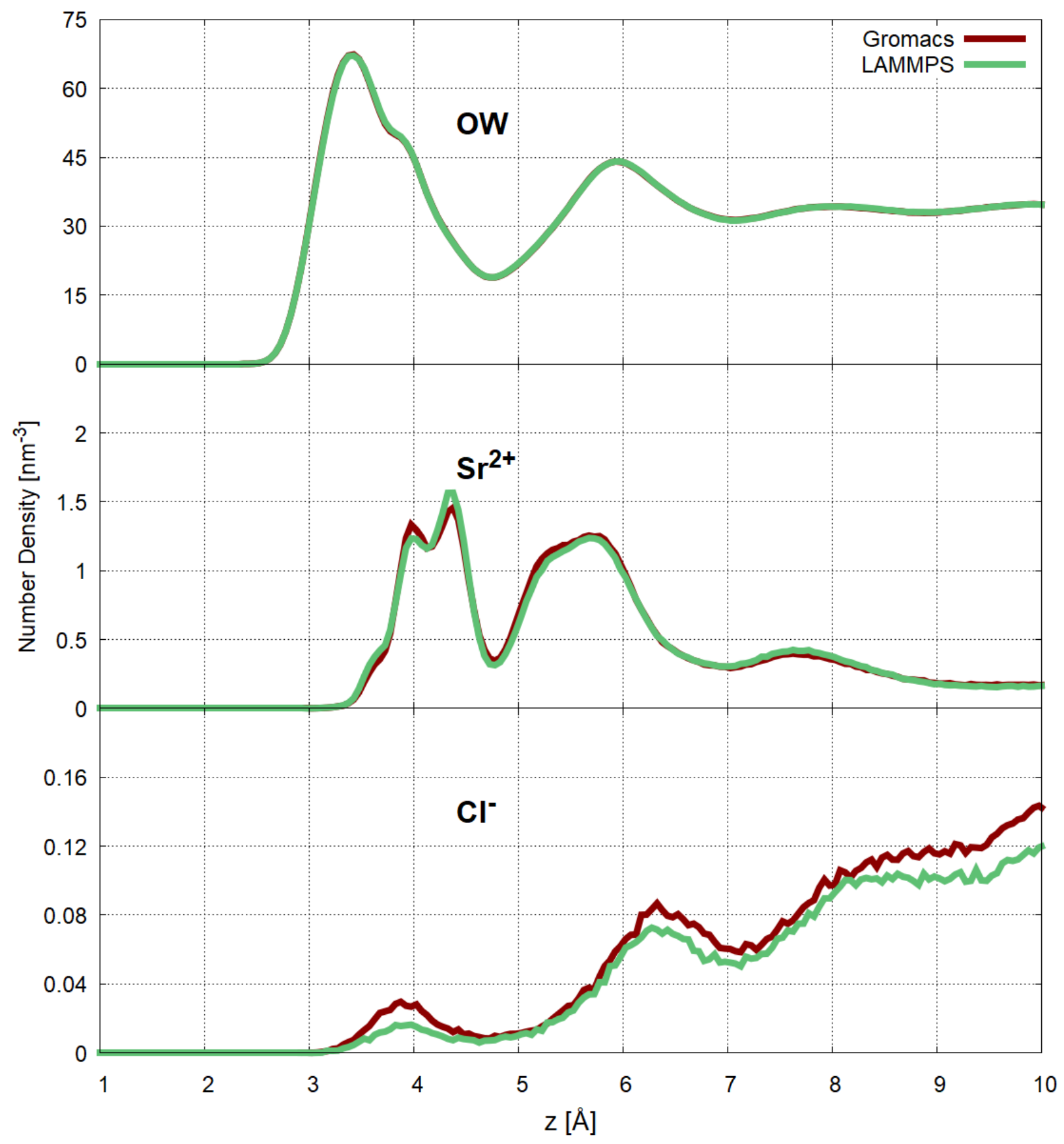

Figure S2. The same as Figure $\mathrm{S} 1$, but for $0.15 \mathrm{M} \mathrm{SrCl}_{2}$ solution. 


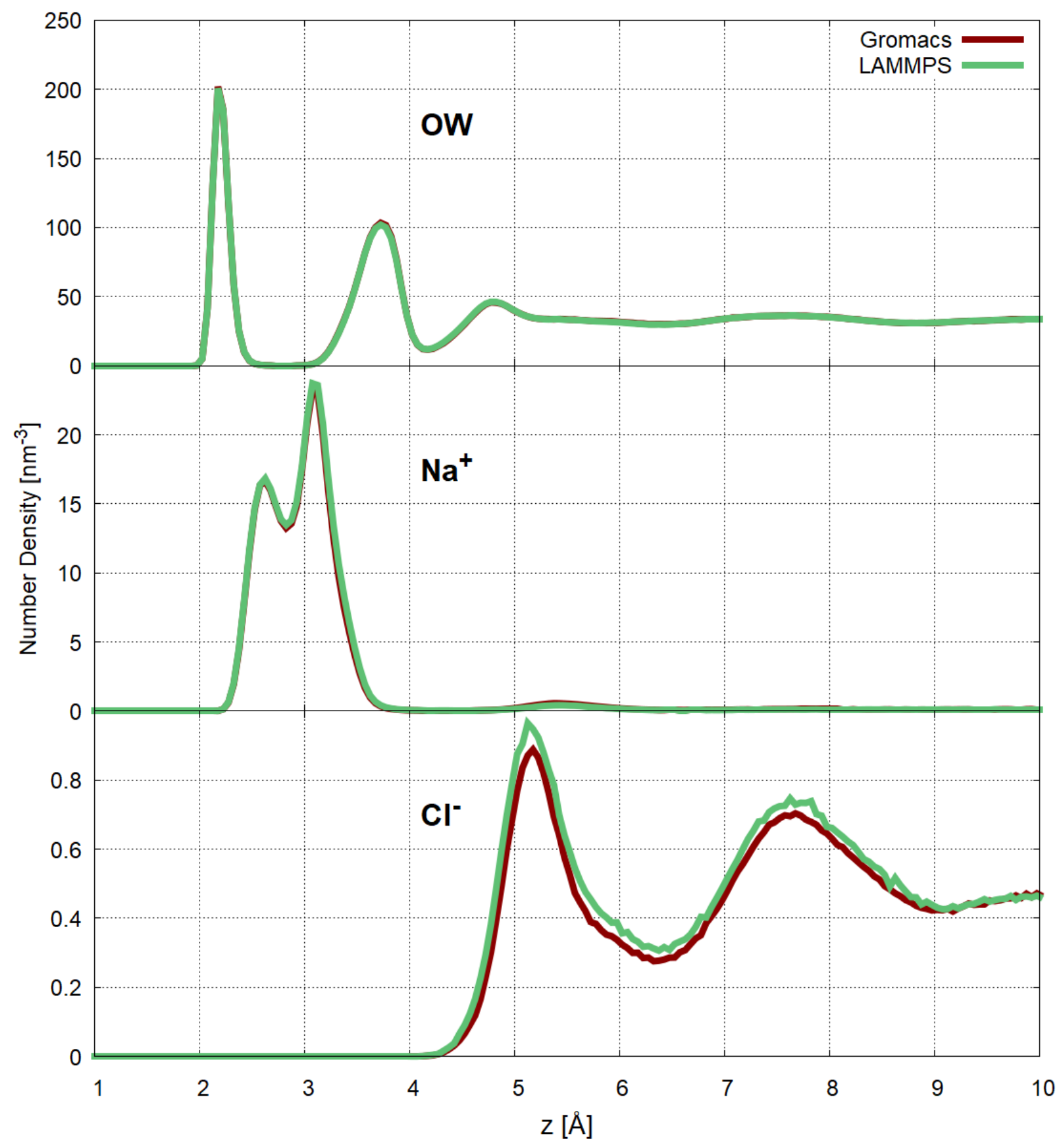

Figure S3. Axial number density profiles of water and ions of $0.45 \mathrm{M} \mathrm{NaCl}$ solution at the negatively charged $\left(-0.208 \mathrm{C} / \mathrm{m}^{2}\right)$ rutile $(110)$ surface from the equilibrium $\mathrm{MD}$ simulations performed in different software packages. Note different vertical scales. 


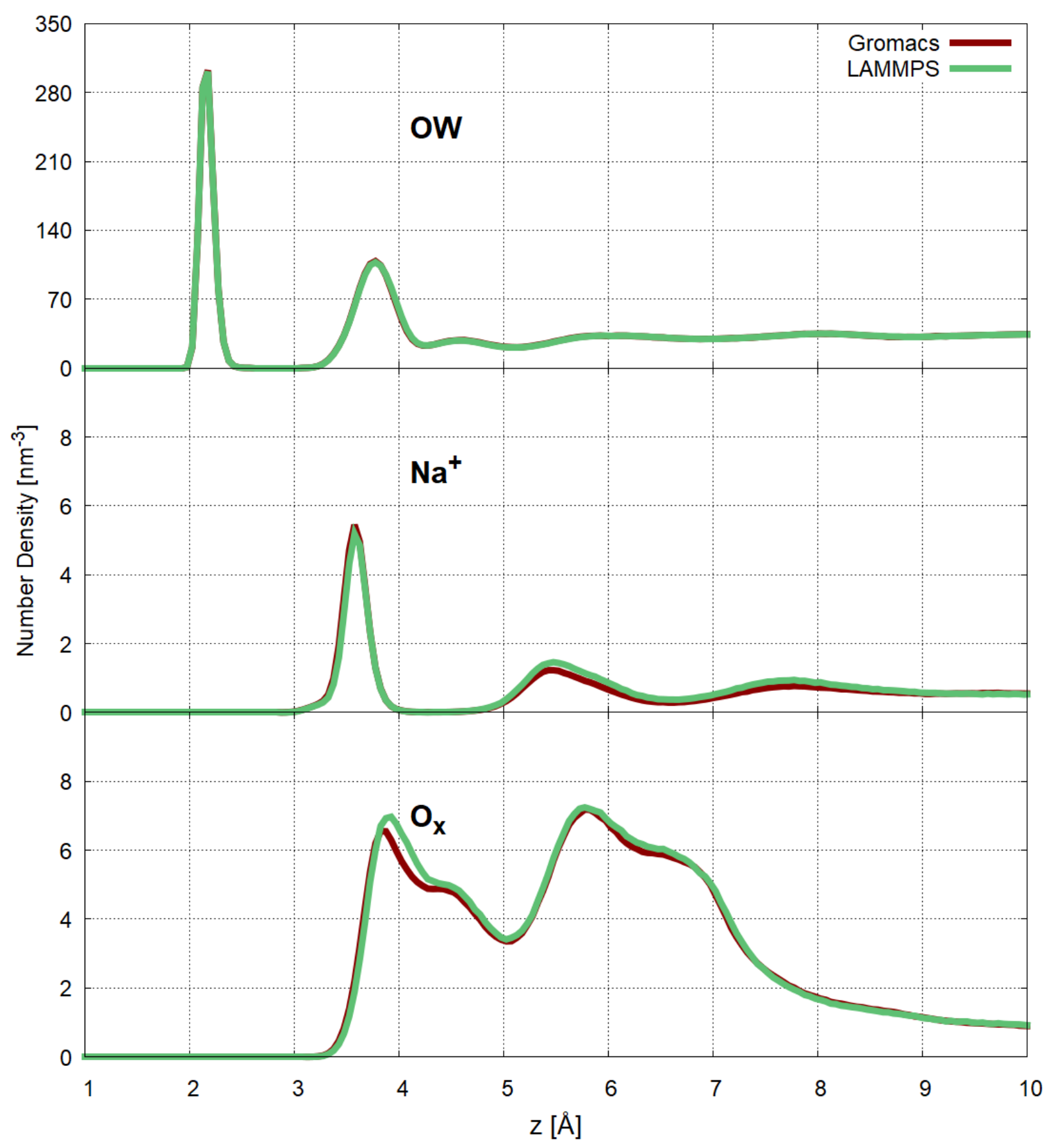

Figure S4. The same as Figure S3, but for $0.29 \mathrm{M} \mathrm{Na}_{2} \mathrm{C}_{2} \mathrm{O}_{4}$ solution at the positively charged $\left(+0.104 \mathrm{C} / \mathrm{m}^{2}\right)$ surface. 
Table S3. Number of Species, Bulk Concentrations, and System Conditions of NEMD

\section{Simulations.}

\begin{tabular}{|c|c|c|c|c|c|c|c|c|c|c|}
\hline $\begin{array}{l}\text { № of } \\
\text { set }\end{array}$ & surface & salt & model & $\begin{array}{c}\text { surface charge } \\
\text { density } \\
{\left[\mathrm{C} / \mathrm{m}^{2}\right]} \\
\end{array}$ & $\mathrm{T}[\mathrm{K}]$ & $\begin{array}{c}\text { \# of } \\
\text { cations }\end{array}$ & $\begin{array}{c}\text { \# of } \\
\text { anions }\end{array}$ & $\begin{array}{l}\text { \# of } \\
\text { water }\end{array}$ & $\begin{array}{c}\text { bulk ionic } \\
\text { concentration } \\
{[\mathrm{M}]} \\
\end{array}$ & figures ${ }^{a}$ \\
\hline \multirow{4}{*}{1} & \multirow{4}{*}{ quartz } & \multirow{4}{*}{$\mathrm{NaCl}$} & \multirow{4}{*}{ ECC } & 0.00 & \multirow{4}{*}{298.15} & 24 & \multirow{4}{*}{24} & \multirow{4}{*}{3745} & 0.37 & \multirow{3}{*}{$5 \mathrm{~A}, 7 \mathrm{~A}$} \\
\hline & & & & -0.03 & & 32 & & & 0.40 & \\
\hline & & & & -0.06 & & 40 & & & 0.45 & \\
\hline & & & & -0.12 & & 56 & & & 0.48 & $5 \mathrm{~A}, 5 \mathrm{~B}, 7 \mathrm{~A}$ \\
\hline \multirow{4}{*}{2} & \multirow{4}{*}{ quartz } & \multirow{4}{*}{$\mathrm{NaCl}$} & \multirow{4}{*}{ ECC } & 0.00 & \multirow{4}{*}{298.15} & 16 & \multirow{4}{*}{16} & \multirow{4}{*}{3745} & 0.25 & $\begin{array}{c}\text { 6, 7A, 7B, S5, S7, } \\
\text { S8 }\end{array}$ \\
\hline & & & & -0.03 & & 24 & & & 0.28 & \multirow{2}{*}{$6,7 \mathrm{~A}, 7 \mathrm{~B}, \mathrm{S5}, \mathrm{S7}$} \\
\hline & & & & -0.06 & & 32 & & & 0.31 & \\
\hline & & & & -0.12 & & 48 & & & 0.33 & $\begin{array}{c}2-4,6,7 \mathrm{~A}, 7 \mathrm{~B} \\
\mathrm{~S} 1, \mathrm{~S} 5, \mathrm{~S} 6, \mathrm{~S} 7, \mathrm{~S} 9\end{array}$ \\
\hline \multirow{4}{*}{3} & \multirow{4}{*}{ quartz } & \multirow{4}{*}{$\mathrm{NaCl}$} & \multirow{4}{*}{ ECC } & 0.00 & \multirow{4}{*}{298.15} & 8 & \multirow{3}{*}{8} & \multirow{4}{*}{3745} & 0.12 & \multirow{4}{*}{$7 \mathrm{~A}$} \\
\hline & & & & -0.03 & & 16 & & & 0.16 & \\
\hline & & & & -0.06 & & 24 & & & 0.16 & \\
\hline & & & & -0.12 & & 36 & 4 & & 0.14 & \\
\hline \multirow{4}{*}{4} & \multirow{4}{*}{ quartz } & \multirow{4}{*}{$\mathrm{NaCl}$} & \multirow{4}{*}{ ECC } & 0.00 & \multirow{4}{*}{298.15} & 4 & 4 & \multirow{4}{*}{3745} & 0.07 & \multirow{4}{*}{$7 \mathrm{~A}$} \\
\hline & & & & -0.03 & & 8 & & & 0.05 & \\
\hline & & & & -0.06 & & 16 & - & & 0.06 & \\
\hline & & & & -0.12 & & 32 & & & 0.07 & \\
\hline & & & & 0.00 & & 16 & & & 0.24 & \\
\hline & & & full- & -0.03 & & 24 & & & 0.26 & \\
\hline & & & quartz & -0.06 & & 32 & & & 0.30 & 4 \\
\hline & & & & -0.12 & & 48 & & & 0.34 & \\
\hline & & & & 0.00 & & 16 & & & 0.26 & \\
\hline & & & & -0.03 & & 24 & 16 & & 0.30 & \\
\hline & & & & -0.06 & & 32 & & & 0.31 & 0 \\
\hline & & & & -0.12 & & 44 & 12 & & 0.29 & \\
\hline
\end{tabular}




\begin{tabular}{|c|c|c|c|c|c|c|c|c|c|c|}
\hline \multirow{4}{*}{6} & \multirow{4}{*}{ quartz } & \multirow{4}{*}{$\mathrm{CaCl}_{2}$} & \multirow{4}{*}{$\mathrm{ECC}$} & 0.00 & \multirow{4}{*}{298.15} & 8 & \multirow{4}{*}{16} & \multirow{4}{*}{3762} & 0.12 & \multirow{4}{*}{6} \\
\hline & & & & -0.03 & & 12 & & & 0.15 & \\
\hline & & & & -0.06 & & 16 & & & 0.14 & \\
\hline & & & & -0.12 & & 24 & & & 0.15 & \\
\hline \multirow{4}{*}{7} & \multirow{4}{*}{ quartz } & \multirow{4}{*}{$\mathrm{SrCl}_{2}$} & \multirow{4}{*}{ ECC } & 0.00 & \multirow{4}{*}{298.15} & 8 & \multirow{4}{*}{16} & \multirow{4}{*}{3762} & 0.13 & $6, S 8$ \\
\hline & & & & -0.03 & & 12 & & & 0.15 & \multirow{2}{*}{6} \\
\hline & & & & -0.06 & & 16 & & & 0.15 & \\
\hline & & & & -0.12 & & 24 & & & 0.15 & 6, S2 \\
\hline \multirow{4}{*}{8} & \multirow{4}{*}{ quartz } & \multirow{4}{*}{$\mathrm{NaCl}$} & \multirow{4}{*}{$\mathrm{ECC}$} & 0.00 & \multirow{4}{*}{373.15} & 16 & \multirow{4}{*}{16} & \multirow{4}{*}{3745} & 0.25 & \multirow{3}{*}{$7 \mathrm{~B}$} \\
\hline & & & & -0.03 & & 24 & & & 0.29 & \\
\hline & & & & -0.06 & & 32 & & & 0.31 & \\
\hline & & & & -0.12 & & 48 & & & 0.33 & $7 \mathrm{~B}, \mathrm{~S} 9$ \\
\hline \multirow{5}{*}{9} & \multirow{5}{*}{ rutile } & \multirow{5}{*}{$\mathrm{NaCl}$} & \multirow{5}{*}{ ECC } & +0.1 & \multirow{5}{*}{298.15} & 11 & 29 & 2182 & 0.41 & \\
\hline & & & & 0.0 & & 17 & 17 & 2200 & 0.42 & \\
\hline & & & & -0.1 & & 34 & 16 & 2186 & 0.38 & $4,5 \mathrm{~A}, 5 \mathrm{~B}$ \\
\hline & & & & -0.2 & & 56 & 20 & 2164 & 0.45 & $4,5 \mathrm{~A}, \mathrm{~S} 3$ \\
\hline & & & & -0.4 & & 94 & 22 & 2128 & 0.39 & $4,5 \mathrm{~A}$ \\
\hline & & & & +0.1 & & 11 & 29 & 2176 & 0.35 & \\
\hline & & & & 0.0 & & 18 & 18 & 2200 & 0.43 & \\
\hline 10 & rutile & $\mathrm{NaCl}$ & full- & -0.1 & 298.15 & 38 & 20 & 2176 & 0.43 & 4 \\
\hline & & & & -0.2 & & 56 & 20 & 2158 & 0.36 & \\
\hline & & & & -0.4 & & 94 & 22 & 2128 & 0.38 & \\
\hline & & & & +0.2 & & 24 & $24,12^{b}$ & 2138 & $0.26^{e}$ & 8 \\
\hline & & & & +0.1 & & 30 & 24 & 2166 & 0.29 & $8, S 4$ \\
\hline 11 & rutile & $\mathrm{Na}_{2} \mathrm{C}_{2} \mathrm{U}_{4}$ & ECC & 0.0 & 298.15 & 24 & 12 & 2204 & 0.28 & \\
\hline & & & & -0.1 & & 54 & 18 & 2162 & 0.31 & 8 \\
\hline & & & & +0.2 & & 5 & $17,7^{b}$ & 2150 & $0.09^{e}$ & \\
\hline 12 & rutile & $\mathrm{Na}_{2} \mathrm{C}_{2} \mathrm{O}_{4}$ & $\mathrm{ECC}$ & +0.1 & 298.15 & 6 & 12 & 2180 & 0.07 & 8 \\
\hline & & & & 0.0 & & 8 & 4 & 2210 & 0.10 & \\
\hline
\end{tabular}




\begin{tabular}{|c|c|c|c|c|c|c|c|c|c|c|}
\hline & & & & -0.1 & & 30 & 6 & 2180 & 0.13 & \\
\hline \multirow{4}{*}{13} & \multirow{4}{*}{ rutile } & \multirow{4}{*}{$\mathrm{Na}_{2} \mathrm{C}_{2} \mathrm{O}_{4}$} & \multirow{4}{*}{ ECC } & +0.2 & \multirow{4}{*}{298.15} & 2 & $16,5,1^{c}$ & 2155 & $0.05(0.02)^{f}$ & \multirow{4}{*}{$\varepsilon$} \\
\hline & & & & +0.1 & & 3 & $10,1^{d}$ & 2185 & $0.05(0.03)^{f}$ & \\
\hline & & & & 0.0 & & 3 & $1,1^{d}$ & 2210 & $0.02(0.03)^{f}$ & \\
\hline & & & & -0.1 & & 21 & $1,1^{d}$ & 2190 & $0.03(0.03)^{f}$ & \\
\hline
\end{tabular}

${ }^{a}$ The last column shows in which figures data from a particular simulation were used. ${ }^{b}$ Number of oxalate and hydrogenoxalate ions, respectively. 'Number of oxalate, hydrogenoxalate, and chloride ions, respectively. ${ }^{d}$ Number of oxalate and chloride ions, respectively. ${ }^{\top}$ Total salt concentration including both (hydrogen)oxalate contributions. In parentheses, the concentration of background $\mathrm{NaCl}$ salt is given. 
Table S4. Technical Settings of Molecular Dynamics Simulations Performed in This Work

\begin{tabular}{|c|c|c|}
\hline setting & Gromacs & LAMMPS \\
\hline timestep $[\mathrm{fs}]$ & \multicolumn{2}{|c|}{2} \\
\hline cut-off $[\AA]$ & \multicolumn{2}{|c|}{ Lorentz-Berthelot } \\
\hline $\begin{array}{c}\text { mixing rules for } \\
\text { interatomic Lennard } \\
\text { Jones interactions }\end{array}$ & Particle Mesh Ewald (PME) & Particle-Particle Particle-Mesh \\
(PPPM)
\end{tabular}




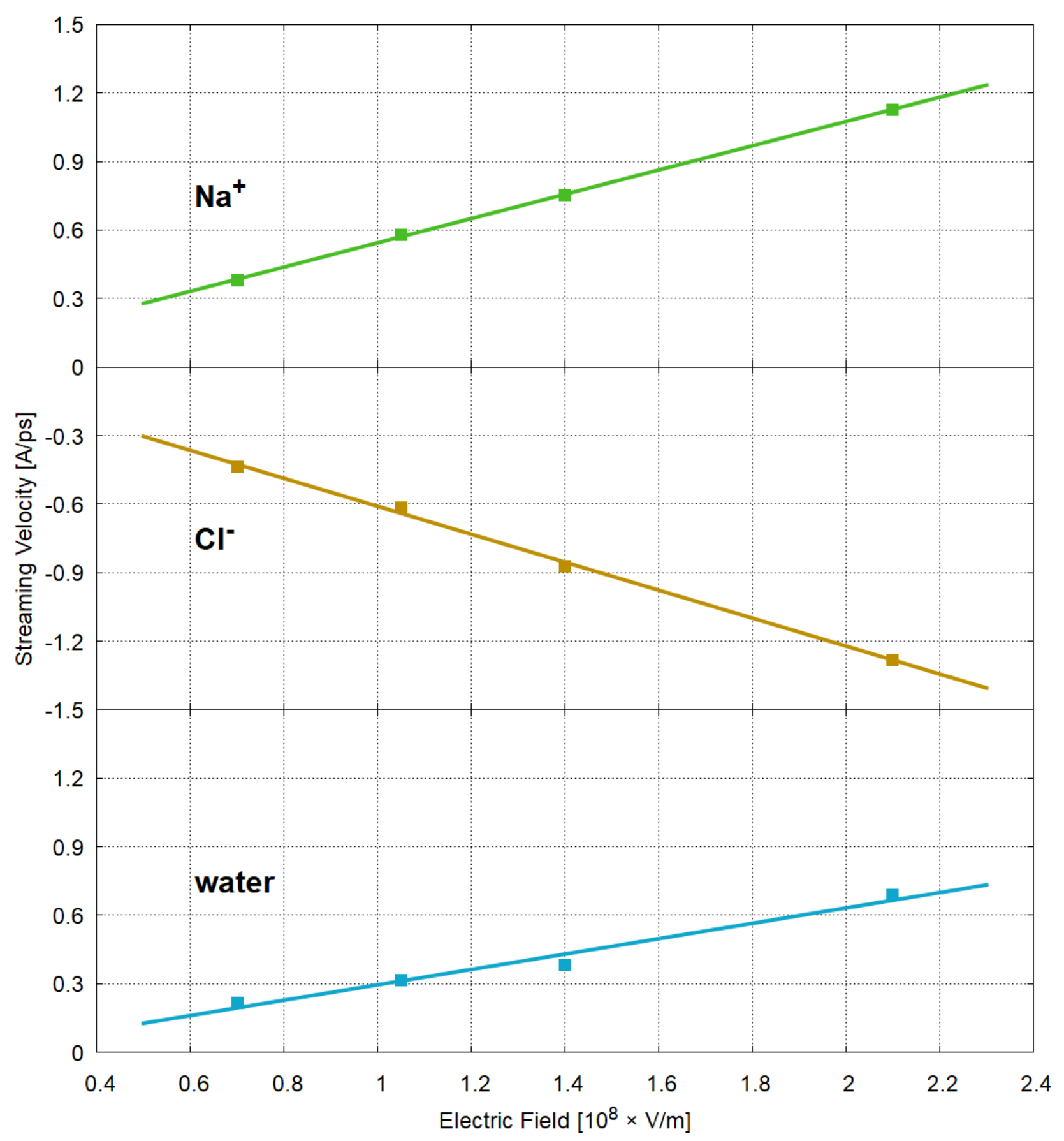

Figure S5. The dependence of streaming velocity as a function of applied electric field for sodium (top), chloride (middle), and water (bottom), for the system with $0.33 \mathrm{M} \mathrm{NaCl}$ solution at the most negatively charged $\left(-0.12 \mathrm{C} / \mathrm{m}^{2}\right)$ quartz $(101)$ surface. Note that for ions we report the velocity with respect to water, while the velocity of water is given relative to the surface. Consequently, the bulk mobility of ions 
does not depend on interfacial structure, while the mobility of water is sensitive to changes at the interface. This explains small deviations in the linear fit for the water velocity, since a too strong electric field can disturb the ionic structure near the surface, and furthermore, affect the relative motion of liquid layers. 

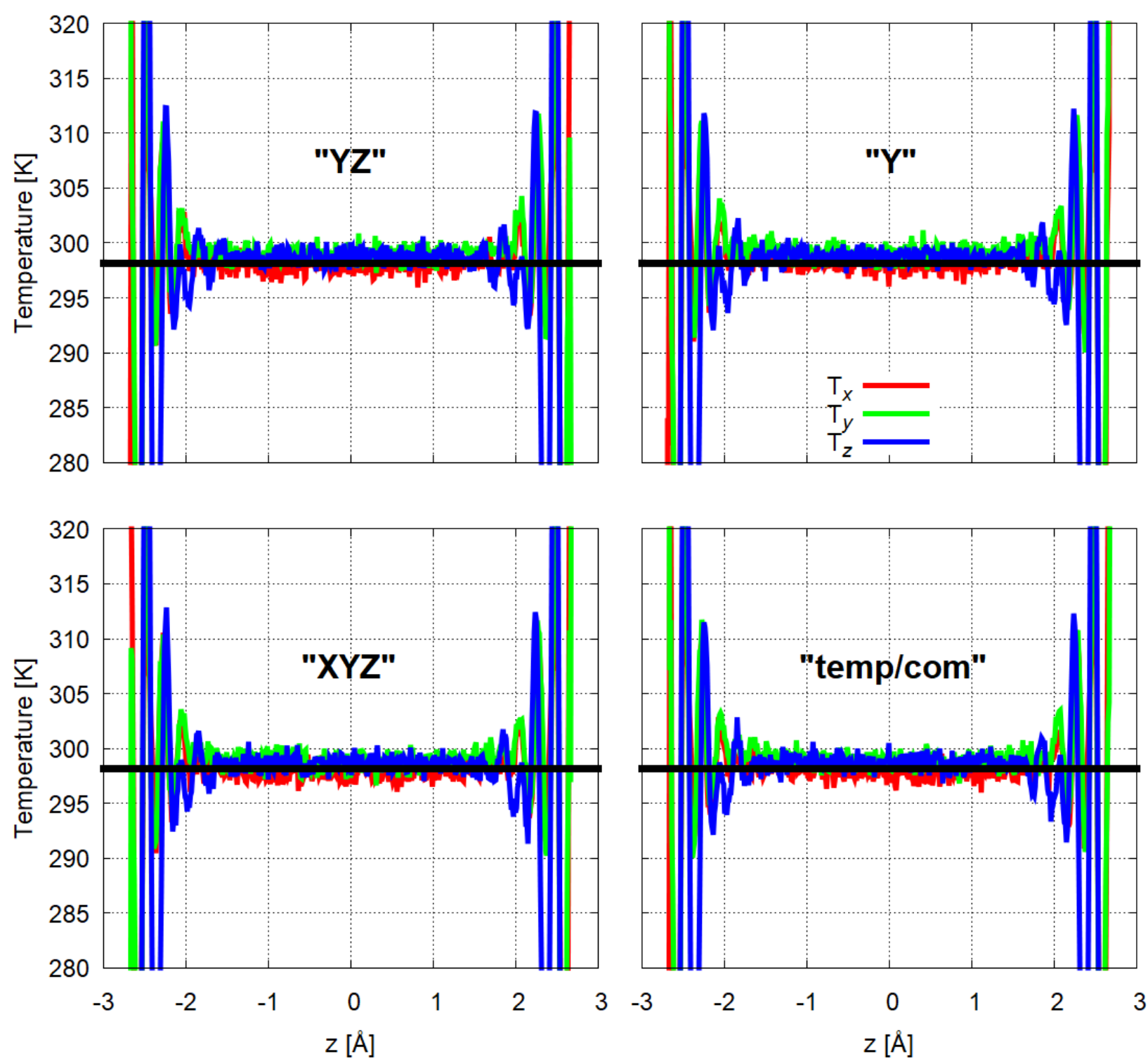

Figure S6. Temperature as a function of $z$-coordinate calculated separately from all velocity components of water and ions at the most negatively charged $\left(-0.12 \mathrm{C} / \mathrm{m}^{2}\right)$ quartz $(101)$ surface in 0.33 $\mathrm{M} \mathrm{NaCl}$ solution, applying different thermostats. Zero is normalized to the center of the simulation box. Huge oscillations in the regions closest to the surfaces (around -2 and $2 \AA$ ) originate from few molecules in very low density domains and do not affect the results. 


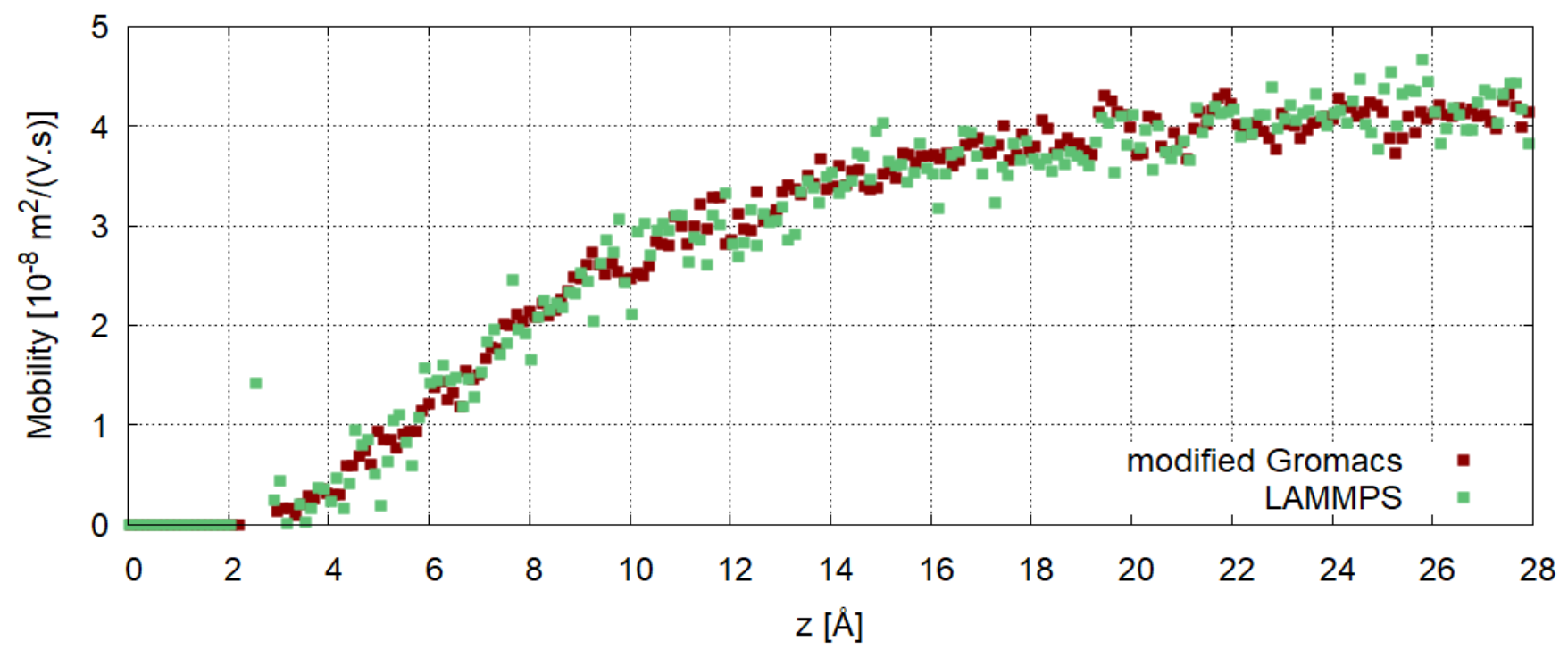

Figure S7. Comparison of distance-dependent mobilities of water at the most negatively charged $\left(-0.12 \mathrm{C} / \mathrm{m}^{2}\right)$ quartz $(101)$ surface in $\mathrm{NaCl}$ solution calculated on-the-fly in LAMMPS simulation or post-processed from trajectories obtained in Gromacs with modified source code implementing the "YZ-thermostat". 


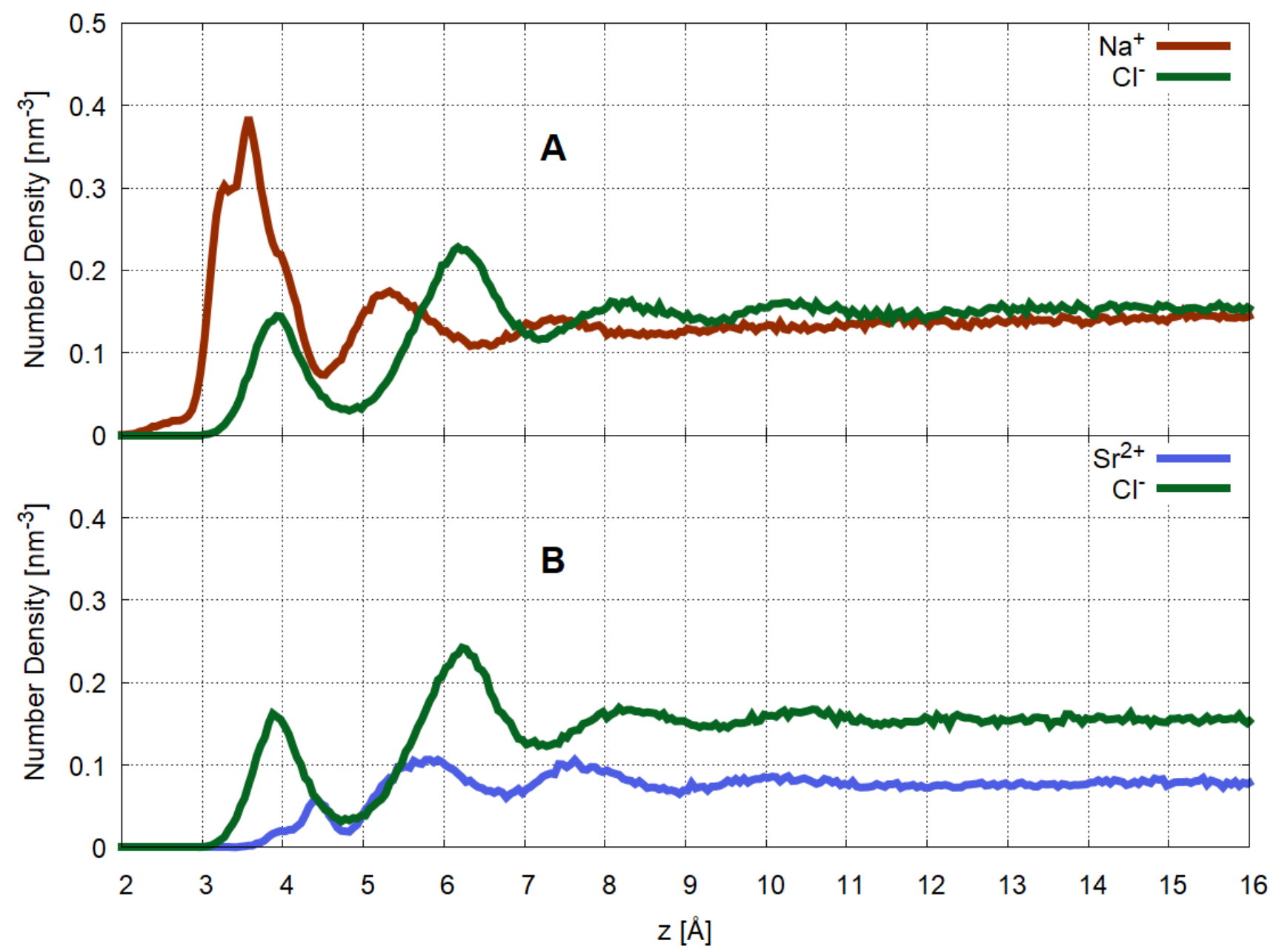

Figure S8. Axial number density profiles of ions at the neutral quartz (101) surface in (A) $0.25 \mathrm{M} \mathrm{NaCl}$ and (B) $0.13 \mathrm{M} \mathrm{SrCl}_{2}$ solutions. 


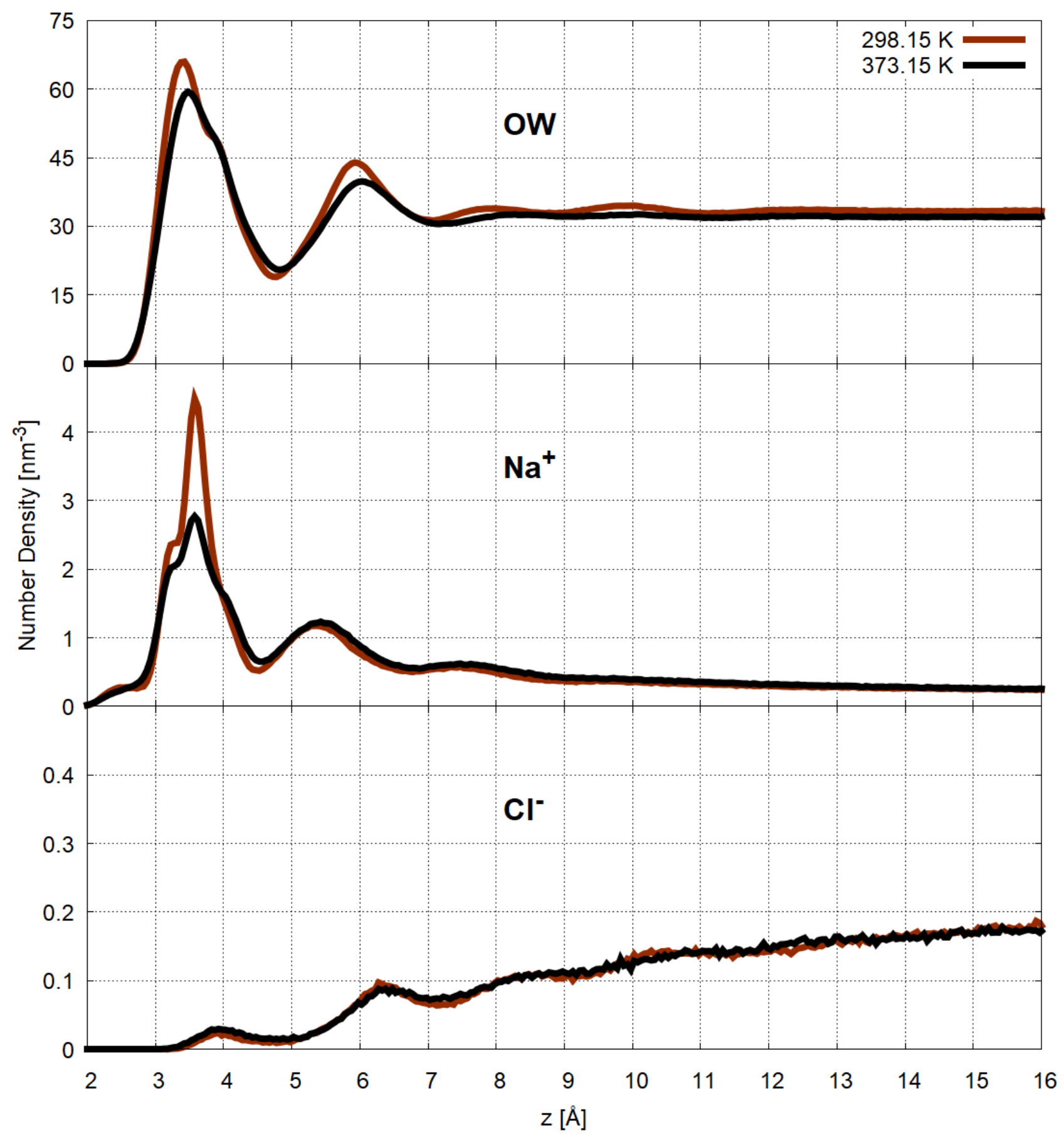

Figure S9. Axial number density profiles of water and ions of $0.33 \mathrm{M} \mathrm{NaCl}$ solution at the most negatively charged $\left(-0.12 \mathrm{C} / \mathrm{m}^{2}\right)$ quartz $(101)$ surface at different temperatures. 


\section{References}

1. Kohagen, M.; Mason, P. E.; Jungwirth, P. Accounting for Electronic Polarization Effects in Aqueous Sodium Chloride via Molecular Dynamics Aided by Neutron Scattering. J. Phys. Chem. B 2015, $120,1454-1460$.

2. Lee, S. H.; Rasaiah, J. C. Molecular Dynamics Simulation of Ion Mobility. 2. Alkali Metal and Halide Ions Using the SPC/E Model for Water at $25^{\circ}$ C. J. Phys. Chem. 1996, 100, 1420-1425.

3. Joung, I. S.; Cheatham, T. E. Determination of Alkali and Halide Monovalent Ion Parameters for Use in Explicitly Solvated Biomolecular Simulations. J. Phys. Chem. B 2008, 112 (30), 9020-9041.

4. Biriukov, D.; Kroutil, O.; Predota, M. Modeling of Solid-Liquid Interfaces using Scaled Charges: Rutile (110) Surfaces. Phys. Chem. Chem. Phys. 2018, 20, 23954-23966.

5. Martinek, T.; Duboué-Dijon, E.; Timr, S.; Mason, P. E.; Baxová, K.; Fischer, H. E.; Schmidt, B.; Pluharova, E.; Jungwirth, P. Calcium Ions in Aqueous Solutions: Accurate Force Field Description Aided by Ab Initio Molecular Dynamics and Neutron Scattering. J. Chem. Phys. 2018, 148, 222813.

6. Pluhařová, E.; Fischer, H. E.; Mason, P. E.; Jungwirth, P. Hydration of the Chloride Ion in Concentrated Aqueous Solutions using Neutron Scattering and Molecular Dynamics. Molecular Physics 2014, 112, 1230-1240.

7. Kroutil, O.; Chval, Z.; Skelton, A. A.; Předota M. Computer Simulations of Quartz (101)-Water Interface over a Range of $\mathrm{pH}$ Values. J. Phys. Chem. C 2015, 119, 9274-9286. 
8. Kroutil, O.; Předota, M.; Kabeláč, M. Force Field Parametrization of Hydrogenoxalate and Oxalate Anions with Scaled Charges. J. Mol. Model. 2017, 23:327. 